\title{
Primary Pulmonary hodgkin's Lymphoma: Two base Reports
}

Aastha Chauhan, Kumud Gupta, Shalini Mullick, Swati Agarwal

From the Department of Pathology, National Institute of Tuberculosis and Respiratory Diseases, Aurobindo Marg, New Delhi- 110030 , India.

\section{Abstract:}

Hodgkin's lymphoma presenting as a primary pulmonary lesion is rare with an incidence of $3.6 \%$ in extranodal lymphomas. It needs to be distinguished from nodal Hodgkin's lymphoma and malignancies secondarily involving the lung. It disseminates in the later stages and involves other lymph nodes and organs early and prompt diagnosis is important. We here present two such cases diagnosed as primary pulmonary Hodgkin's lymphoma in young adult females who presented with nonspecific complaints.

Key words: Hodgkin's Disease, Nervous System Neoplasms, Humans, Lymph Nodes, Incidence.

\section{Introduction}

Primary pulmonary Hodgkin's lymphoma is a very rare neoplasm. Fewer than 100 cases have been reported in the literature so far [1]. It is a discrete entity which needs to be distinguished from nodal Hodgkin's disease and from lymphomas secondarily involving lung [2]. We hereby present two such cases of pulmonary Hodgkin's lymphoma diagnosed on histopathology.

\section{Case Reports}

Two young adult female patients presented with chief complaints of chest pain and cough with foul smelling expectoration since 2 years. Patients also complained of low grade fever and breathlessness since 4 months. On physical examination there was no peripheral lymphadenopathy and organomegaly in both patients. Biochemistry, blood culture and urine microscopy were normal in both patients. Pulmonary function tests were satisfactory.

\section{Case 1}

28 year old female presented with history of left sided chest pain, cough and fever. Hematological investigations revealed a hemoglobin of $9 \mathrm{~g} /$ $\mathrm{dL}$, a total leucocyte count of $16,600 / \mathrm{mm}^{3}$ with neutrophilic leucocytosis. Peripheral smear showed no atypical cells. Ultrasound chest showed a hypodense lesion with air and debris measuring $7.8 \times 5.2 \mathrm{cms}$ in the left upper lobe of lung. CT scan chest showed a thick walled cavitatory lesion measuring $10 \times 10 \times 7 \mathrm{cms}$ in left upper lobe with areas of necrosis suggesting a malignant lesion. The lesion was not reaching upto the pleura. Minimal pleural and pericardial effusion was also noted.

\section{Corresponding Author: Dr. Shalini Mullick}

Email: shalini.mullick@gmail.com

Received: June 24, 2014 | Accepted: August 9, 2014 | Published Online: September 15, 2014

This is an Open Access article distributed under the terms of the Creative Commons Attribution License (creativecommons.org/licenses/by/3.0)

Conflict of interest: None declared | Source of funding: Nil | DOI: http://dx.doi.org/10.17659/01.2014.0088 
Flexible bronchoscopy showed a normal airway. Bronchial washings, pleural fluid and sputum cytology were negative for malignant cells. ZN staining for AFB was negative. Lung biopsy from the mass lesion showed mainly necrosis and occasional large atypical cells. No conclusive diagnosis was made. Following which left sided pneumectomy was done. Resected left lung showed a greyish white tumor mass measuring $8 \times 7 \times 4 \mathrm{cms}$ with necrosis and cavitation [Fig.1]. The margins were not well demarcated from the adjacent lung parenchyma. Left main bronchus and left lower lobe were unremarkable grossly. No enlarged lymph-nodes were identified.

\section{Case 2}

A 26 year old female presented with chief complaints of cough with expectoration and low grade fever. Hematological investigations revealed a haemoglobin of $11.1 \mathrm{~g} / \mathrm{dL}$, a total leucocyte count of $13,400 / \mathrm{mm}^{3}$ with neutrophilic leucocytosis. Peripheral smear showed no atypical cells. CT thorax showed a mass lesion measuring approximately $8 \times 6 \times 5 \mathrm{cms}$ involving right upper lobe and middle lobe with mediastinal lymphadenopathy [Fig.2]. Pleural and pericardial effusion was noted. Flexible bronchoscopy showed a normal airway. Bronchial washings, pleural fluid and sputum cytology were negative for malignant cells. Open lung biopsy was done. Two grey brown soft tissue pieces measuring $1.5 \times .6 \times .3 \mathrm{cms}$ and $1 \times 1 \times .5 \mathrm{cms}$ from each right upper lobe and middle lobe were sent for histopathological examination.

The microscopic features were similar in both the cases. Sections showed typical Reed-Sternberg cells ( $R-S$ cells) along with mononuclear, multinucleate tumor cells with prominent nucleoli in a polymorphous background comprising many eosinophils, few neutrophils and plasma cells [Fig.3]. Areas of necrosis along with occasional mitotic figures were noted. In case 2 features of obstructive pneumonia

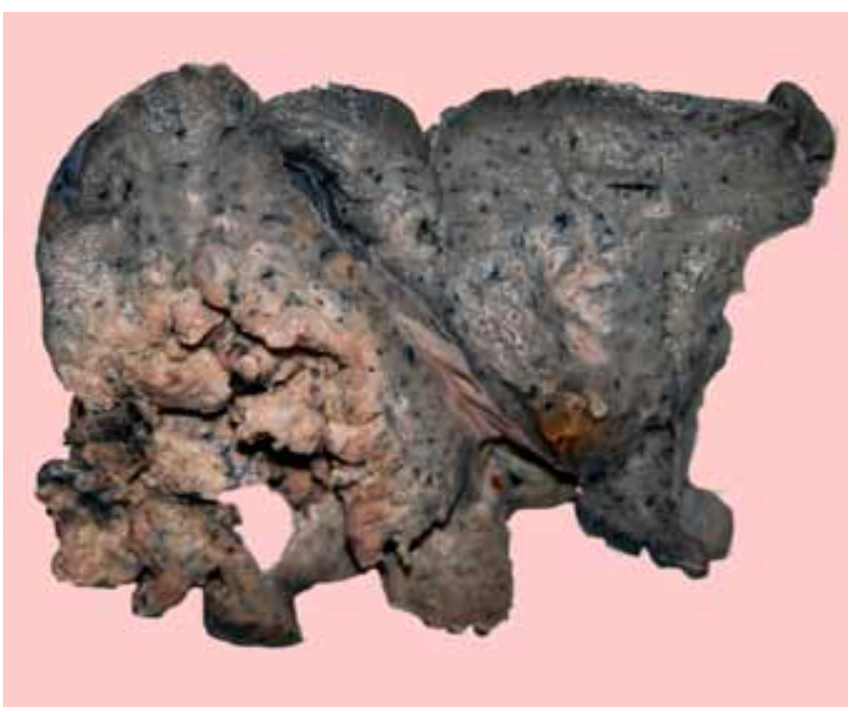

Fig. 1: A necrotic cavitory tumor mass measuring $8 \times 7 \times 4 \mathrm{cms}$ in the upper lobe of the left lung (Case 1).

were also noted. On immunohistochemistry, ReedSternberg cells and mononuclear cells expressed CD15 and CD30 positivity and CD45 negativity in both the cases [Fig.4]. A final diagnosis of primary pulmonary classical Hodgkin's lymphoma ( $\mathrm{CHL}$ ) was rendered in both the cases. In case 1, patient is under clinical follow up following surgery and chemotherapy and has not relapsed. In case 2, patient is responding well to chemotherapy.

\section{Discussion}

Primary pulmonary Hodgkin's disease is a rare entity. Less than 100 cases have been reported in the literature so far [1]. It has a bimodal age distribution with peak incidence being in the third and sixth/seventh decade and a female predominance [3]. A primary origin in the lung can be explained by the significant amount of lymphoid tissue in the peri-bronchial areas [4].

Kern et al. [4] suggested the following criteria for diagnosing the primary pulmonary Hodgkin's disease (CHL): (i) diagnosis by pathological 


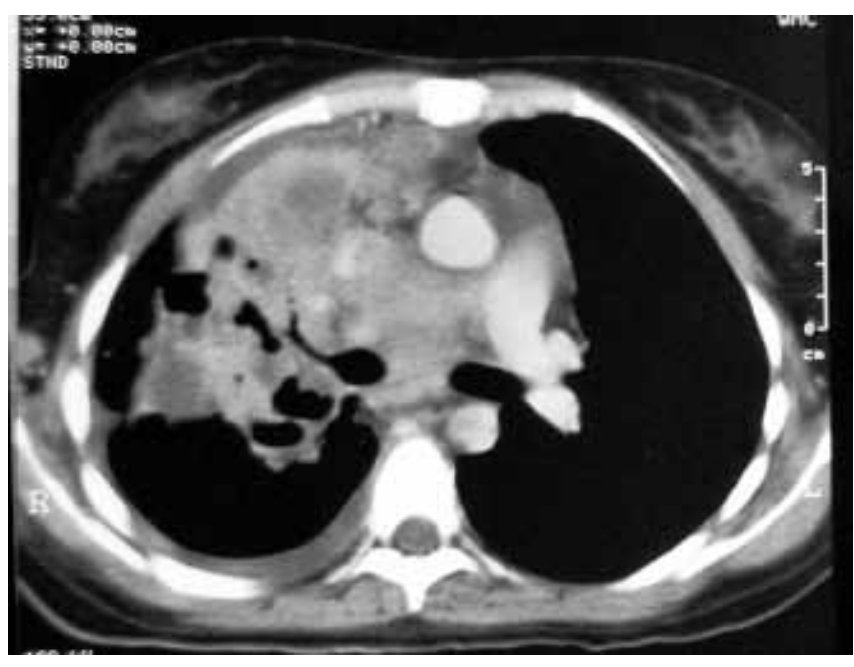

Fig.2: CT scan of the chest showing a mass lesion in the right lung involving upper and middle lobe with areas of necrosis with mediastinal lymphadenopathy (Case 2).

examination documented by photomicrographs or microscopy, (ii) disease predominantly in the lung at the time of the original diagnosis, with or without simultaneous involvement of hilar or mediastinal, lymph nodes, and (iii) exclusion of cases with evidence of peripheral or other distant lymph node or organ involvement and cases in which pulmonary tumor appears to be an extension of a mediastinal lesion. In our cases, the tumor was predominantly in the lung with mediastinal lymph node enlargement in case 2. There was no evidence of disease at other sites at the time of initial presentation.

Pulmonary Hodgkin's disease can present as a solitary mass, cavitory lesion or diffuse involvement of the lungs [5]. The most common clinical presentation is dry cough. Due to the non-specific presentation of the disease, non-invasive tests are often noncontributory. Sputum cytology is rarely positive in cases in which intraluminal growth or mucosal ulceration promotes the exfoliation of diagnostic cells in sputum and bronchoscopy with bronchoalveolar lavage is unrevealing. However, bronchoscopy is recommended to exclude the other

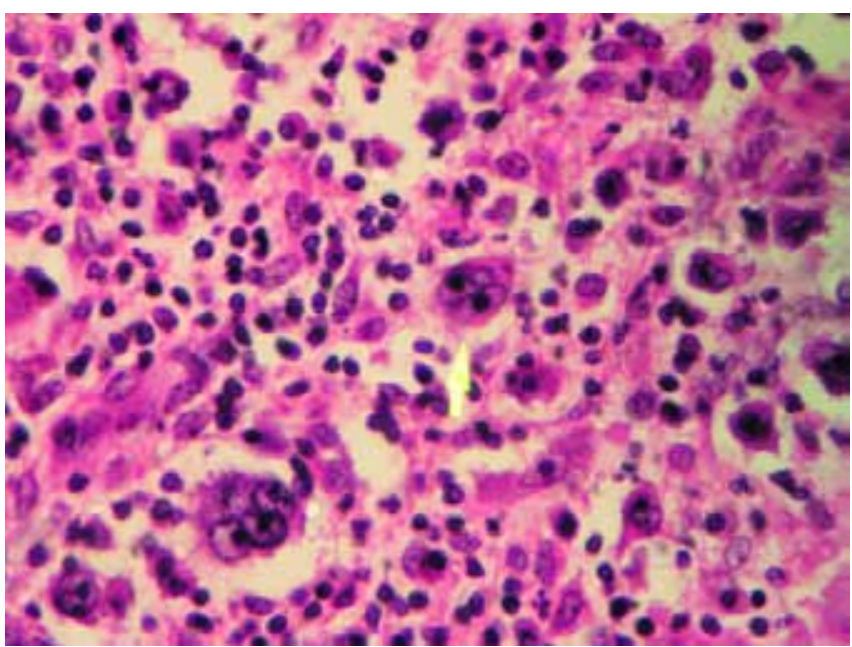

Fig.3: Classical Reed-Sternberg cell (arrow) and mononuclear and multinucleate tumor cells in a mixed inflammatory cell background ( $H \& E$ staining $x 400$ ) (Case 1).

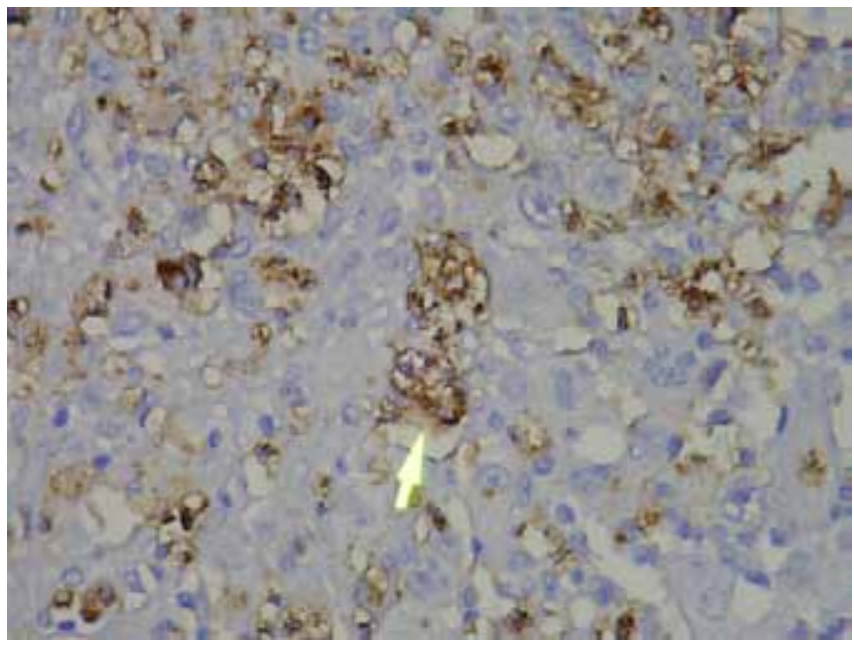

Fig.4: Immunostaining for CDI5 positive in cytoplasm of multinucleate tumor cell (400X) (Case 1).

infectious and neoplastic disorders [6]. Boshnakova et al. reported that bronchoscopic biopsies were inconclusive in their two cases with endobronchial involvement, due to lack of Reed-Sternberg cells [7]. Definitive diagnosis often requires open thoracotomy and lung biopsy. 
Histopathologically, nodular sclerosis is the most common subtype followed by mixed cellularity. The differential diagnosis includes tuberculous and fungal granulomas, Langerhans histiocytosis, metastatic carcinomas and Non-Hodgkin's lymphoma mainly $T$ cell lymphomas [7]. Undifferentiated carcinomas may show the presence of Reed-Sternberg like cells, but neutrophils are frequently seen and eosinophils are seldom present [8]. In difficult cases immunohistochemistry resolves the diagnostic problem. Reed-Sternberg cells and its variants are CD15 and CD30 positive [9].

The treatment options include surgery in localized tumors, if the lesions are diffuse or involve both lungs chemotherapy and radiotherapy are given [10]. Negative prognostic factors include the involvement of both lungs, pleura or more than one lobe, cavitation, advanced age, multiple lesions and general symptoms [7]. Two-year survival rate without relapse is observed in less than $50 \%$ of the patients [3].

\section{Conclusion}

A preoperative diagnosis of pulmonary Hodgkin's disease with an immunohistochemistry panel in combination with imaging offers a chance to the patient to be given specific treatment thus improving prognosis and preventing spread of the disease. This entity inspite of its rare incidence has to be kept in mind as differential diagnosis while evaluating neoplastic pulmonary lesions.

\section{References}

1. Homma M, Yamochi-Onizuka T, Shiozawa
E, Takimoto M, Ariizumi $H$, Nakashima $H$ et al. Primary Pulmonary Classical Hodgkin's Lymphoma with Two Recurrences in the Mediastinum: A Case Report. J Clin Exp Hematopathol 2010;50(2):151-157.

2. Pai RR, Raghuveer $C V$, Philipose RT, Shetty $A B$. Primary Pulmonary Hodgkin's Disease: A Distinct Entity. Indian J Chest Allied Sci 2006;48:139141.

3. Stachura T, Malinowski E. Primary Pulmonary Hodgkin's Lymphoma with Epstein-Barr and Cytomegaly Virus Infections. A Case Report and Differential Diagnosis. Pol J Pathol 2003;54(1):79-83.

4. Kern WH, Crepeau AG, Jones JC. Primary Hodgkin's disease of the lung. Report of 4 cases and review of the literature. Cancer $1961 ; 14: 1151-1165$.

5. Cartier Y, Johkoh T, Honda O, Muller NL. Primary Pulmonary Hodgkin's disease: CT findings in three patients. Clin Radiol 1999;54:182-184.

6. Harper PG, Fisher C, Mclennan K, Souhami RL. Presentation of Hodgkin's disease as an endobronchial lesion. Cancer 1984;53:147150.

7. Radin Al. Primary Pulmonary Hodgkin's disease. Cancer 1990;65:550-563.

8. Boshnakova T, Michailova V, Koss M, Georgiev C, Todorov T, Sabrinova M. Primary Pulmonary Hodgkin,s disease - Report of two cases. Respir Med 2000;94:830-831.

9. Corrin B. Lymphoproliferative disease. In: Corrin B, editor. Pathology of the Lungs. 1 st ed. London: Churchill Livingstone:2000; pp.555-572.

10. Cadranel J, Wislez $M$, Antoine M. Primary Pulmonary Lymphoma. Eur Respir J 2002;20:750-762. 